\title{
Upsurge of caesarean sections in Pakistan: A qualitative study on perceptions of women and gynaecologists
}

\section{Aaisha Amjad}

University of the Punjab

\section{Ahmed Usman}

University of the Punjab

Fauzia Saleem Alvi

University of the Punjab

Muhammad Farooq

University of Central Punjab

Nabiha Shahram

University of the Punjab

Aisha Jalil

University of Lahore

Florian Fischer ( $\nabla$ florian.fischer@rwu.de )

Ravensburg-Weingarten University of Applied Sciences https://orcid.org/0000-0002-4388-1245

Research article

Keywords: childbirth, caesarean section, pregnancy, culture, fear

Posted Date: June 24th, 2020

DOI: https://doi.org/10.21203/rs.3.rs-34756/v1

License: (9) (i) This work is licensed under a Creative Commons Attribution 4.0 International License.

Read Full License 


\section{Abstract}

Background: The upsurge of caesarean sections in Pakistan has become a matter of concern for public health. The current rate of caesarean sections has reached $19.6 \%$ of total childbirths in Pakistan. This study attempts to explore the perceptions of married women and gynaecologists about different social and cultural aspects affecting the upsurge of caesarean sections in Pakistan.

Methods: In-depth interviews were conducted with pregnant women, women who already have given birth via caesarean section and gynaecologists in the leading public and private maternity hospitals located in Lahore, Pakistan. Qualitative data collected was analysed using thematic content analysis technique.

Results: Major findings of the study revealed that the personal experience of previous caesarean section, experiences shared by other women, caesarean sections as a family trend, caesarean sections as a status symbol, fears related to vaginal childbirth, and lack of awareness about modes of childbirth are the most significant social and cultural aspects contributing to the upsurge of caesarean sections in Pakistan.

Conclusion: Caesarean sections as a symbol of higher socio-economic status and the fear of prolonged labour during vaginal childbirth are the two key reasons to prefer caesarean sections. There is a need to create awareness among women about coping skills to deal with the fear and anxiety related to vaginal birth in order to improve the overall maternal health in Pakistan.

\section{Background}

Pregnancy and childbirth are considered as important events in the life of a woman. However, in some cases, the childbirth process might turn into a stressful experience for a woman if it involves medical complications resulting into caesarean section surgery [1]. Along with medical reasons, the belief system, behavioural patterns, and lack of awareness among women also affect their decision regarding the mode of childbirth [2]. Several studies reveal that psychological, social and cultural factors tend to influence the perceptions of women about pregnancy and childbirth process [2, 3]. Caesarean sections have become more of a social issue since a significant number of pregnant women have started to choose caesarean sections even without medical indications, resulting in its upsurge worldwide [4]. This increase has attracted the attention of experts in the field of public health globally. Likewise, assessing the gravity of this issue, the World Health Organization recommends that the caesarean section rate should not exceed $15 \%$ in any country across the globe [5].

Caesarean section rate has been varying in different parts of the world. In many countries, the rate of caesarean sections has reached $25 \%$ [6]. Likewise, most recent data indicate that the current rate of caesarean sections is $19.6 \%$ in Pakistan. For that reason, Pakistan is amongst those developing countries that are responsible for the worldwide increase in caesarean sections [7]. Despite certain side effects and post-delivery complications, caesarean sections are emerging as the first choice for childbearing worldwide [6]. 
There can be several factors behind the upsurge of caesarean sections including the greater involvement of medical technology to overcome childbirth complications [8]. It has been observed that the medicalisation of childbirth has also increased the concerns regarding the malpractices associated with the use of medical technology by the doctors, who may prefer an unnecessary caesarean surgery for their convenience, quick handling to save time or economic incentives [9]. Additionally, the inadequate knowledge of pregnant women about delivery complications tends to increase the likelihood of their exploitation for economic gain by the medical staff [10]. In such cases, it becomes easy for physicians and hospital authorities to persuade women and their families to opt for caesarean sections by associating the method with the security of mother and child health, even when the baby could be delivered normally $[9,10]$.

The research studies conducted with an aim to understand the preference of caesarean sections over vaginal birth highlight multiple factors including request by pregnant woman, women's age at the time of childbirth, expectancy of twins or multiple foetus, induced labour, breach presentation of baby, and previous history of caesarean section $[11,12]$. Additionally, fear of labour pain, physical impact of vaginal delivery, convenience of delivery in an estimated and certain time, and decreased risks related to neonatal and pelvic floor are considered as other significant reasons for the preference of caesarean sections over vaginal births [13]. In most of the countries, women are given a choice to opt either for vaginal or caesarean delivery. Thus, in order to elect the mode of delivery, women experience a subjective decisionmaking process influenced by several factors [14]. Culture, societal perceptions, personal insights, and awareness collectively influence the decision-making process and preferences of women in a society [15]. Similarly, the role of culture has a significant impact on women's perceptions toward pregnancy, childbirth process, and preference of delivery mode [16].

Despite this backdrop, there is a paucity of research about pregnant women's preferential patterns related to caesarean sections. There is scarcity of research studies in Pakistan to examine the perceptions of women and gynaecologists regarding social and cultural factors contributing to the upsurge of caesarean sections. Therefore, the present study aims to examine social and cultural aspects highlighted by women who are either currently pregnant or delivered via caesarean section and gynaecologists that influence the preference of caesarean section over vaginal childbirth in the Pakistani context.

\section{Methods}

\section{Study design}

We used a qualitative study design to understand the perceptions of pregnant and delivered women as well as gynaecologists regarding the upsurge of caesarean sections in Pakistan. This research approach supports to describe the acquired patterns of behaviours and prevalent beliefs and values of a cultural group [17]. Qualitative research also considers culture as a lens for understanding participants' views and perceptions about social realities [18]. An interpretive approach in order to gain a comprehensive and rigorous understanding of the topic has been employed [17]. An interpretive theory is generated based on 
the data collected from pregnant and delivered women and gynaecologists in the leading maternity hospitals in Lahore, a city in Pakistan.

A study found that the rate of caesarean sections showed an increase in both the private and the public sector hospitals in Pakistan without significant variation [10]. Keeping this in view, we purposively selected renowned private sector hospitals (i.e. Hameed Latif Hospital and National Defence Hospital) and public sector hospitals (i.e. Sheikh Zayed Hospital and Services Hospital) in Lahore considering their reputation in imparting the modern maternity services and mother-child care. Data were collected between May and September 2019.

\section{Sample}

The participants of the present study consisted of 15 female gynaecologists, 10 pregnant women who visited the hospitals to receive antenatal care, and 10 women who had caesarean section and visited the hospital to receive postnatal care. The common characteristics of the pregnant women included: (a) They had a previous caesarean section, (b) they were in the third trimester of pregnancy, (c) they received regular antenatal care, and (d) they had no pregnancy complications. The common characteristics of delivered women were: (a) They had at least one caesarean section previously, (b) they all had caesarean section at the last childbirth, and (c) they visited the hospital for postnatal care. The mean age for all women was 29 years, with a range from 20 to 38 years (Table 1 ). 
Table 1

Demographic characteristics of pregnant and delivered women $(n=$ 20)

\begin{tabular}{|c|c|c|c|}
\hline No. & $\begin{array}{l}\text { Age range } \\
\text { (in years) }\end{array}$ & Type of women & Hospital visited/admitted \\
\hline 1 & $20-29$ & Pregnant & Hameed Latif Hospital \\
\hline 2 & $20-29$ & Pregnant & National Defence Hospital \\
\hline 3 & $30-39$ & Delivered & Services Hospital \\
\hline 4 & $20-29$ & Pregnant & Sheikh Zayed Hospital \\
\hline 5 & $30-39$ & Pregnant & Hameed Latif Hospital \\
\hline 6 & $30-39$ & Delivered & Services Hospital \\
\hline 7 & $30-39$ & Pregnant & Services Hospital \\
\hline 8 & $20-29$ & Delivered & Hameed Latif Hospital \\
\hline 9 & $20-29$ & Delivered & Sheikh Zayed Hospital \\
\hline 10 & $20-29$ & Delivered & Sheikh Zayed Hospital \\
\hline 11 & $30-39$ & Pregnant & Hameed Latif Hospital \\
\hline 12 & $30-39$ & Delivered & Sheikh Zayed Hospital \\
\hline 13 & $20-29$ & Pregnant & National Defence Hospital \\
\hline 14 & $20-29$ & Delivered & Sheikh Zayed Hospital \\
\hline 15 & $30-39$ & Delivered & National Defence Hospital \\
\hline 16 & $30-39$ & Pregnant & Sheikh Zayed Hospital \\
\hline 17 & $20-29$ & Delivered & Services Hospital \\
\hline 18 & $20-29$ & Pregnant & National Defence Hospital \\
\hline 19 & $30-39$ & Pregnant & Services Hospital \\
\hline 20 & $20-29$ & Delivered & Hameed Latif Hospital \\
\hline
\end{tabular}

Out of female gynaecologists, four were practicing in Hameed Latif Hospital, four were selected from National Defense Hospital, four were senior gynaecologists in Sheikh Zayed Hospital, and three were serving in Services Hospital. The gynaecologists were selected employing purposive sampling technique by selecting those who were renowned for performing successful child deliveries (both vaginal and caesarean) and had the experience of at least five years (Table 2). 
Table 2

Demographic characteristics of the gynaecologists $(n=15)$

\begin{tabular}{|c|c|c|c|}
\hline No. & $\begin{array}{l}\text { Age range } \\
\text { (in years) }\end{array}$ & Hospital & Practice experience (in years) \\
\hline 1 & $50-59$ & Hameed Latif Hospital & 6 \\
\hline 2 & $40-49$ & National Defence Hospital & 8 \\
\hline 3 & $50-59$ & Services Hospital & 9 \\
\hline 4 & $40-49$ & Hameed Latif Hospital & 5 \\
\hline 5 & $40-49$ & National Defence Hospital & 8 \\
\hline 6 & $50-59$ & Services Hospital & 8 \\
\hline 7 & $50-59$ & Sheikh Zayed Hospital & 5 \\
\hline 8 & $40-49$ & Hameed Latif Hospital & 13 \\
\hline 9 & $50-59$ & National Defence Hospital & 9 \\
\hline 10 & $50-59$ & Services Hospital & 11 \\
\hline 11 & $40-49$ & Hameed Latif Hospital & 10 \\
\hline 12 & $50-59$ & National Defence Hospital & 8 \\
\hline 13 & $50-59$ & Sheikh Zayed Hospital & 15 \\
\hline 14 & $50-59$ & Sheikh Zayed Hospital & 14 \\
\hline 15 & $40-49$ & Sheikh Zayed Hospital & 12 \\
\hline
\end{tabular}

\section{Data collection}

All the participants voluntarily participated in the study and were interviewed in the hospital setting. In the first meeting, the researchers introduced themselves and each participant was informed about the purpose of the study. The participants were assured about the confidentiality of the personal information. After having their written consent, the time for the interview was mutually decided. The interviews with gynaecologists were conducted in their offices and women participants were interviewed in vacant rooms in the respective hospitals. The average time for each interview was around 45 to 90 minutes.

An interview guide was developed for this study and used for conducting in-depth interviews (see Appendix). After a detailed rapport building session, the individual participant was asked to reflect on the social and cultural aspects affecting the increase of caesarean sections in the Pakistani context. Some probing questions were also part of the interviews and were asked where additional detailed information was needed. At the end of each interview, the participant was encouraged to express her views which she felt could not be described during the interview. Demographic characteristics of the participants and the 
information related to the pregnancy and mode of delivery was also obtained. All of the interviews were audio-recorded and transcribed afterwards. Data saturation was achieved after 30 interviews when no new idea about caesarean section was shared by the participants. The transcribed interviews were given to each participant for the correction of information shared by them.

\section{Data analysis}

All of the interviews were transcribed verbatim after conducting an interview. As the data analysis was to be carried out in English, all the interviews were translated from Urdu to English language. All interviews were translated through Express Scribe software. Data was anonymised by detaching all the identifiers from the data in order to ensure anonymity. All the transcribed and translated transcripts were used for a thematic content analysis. We identified codes based on the ideas expressed by the study participants in form of inductive codes and in addition further deductive ones. A code book was developed that provided a central reference for coding all transcripts. After coding the transcripts, the coded data was categorised by grouping together the similar codes that led to identifying major themes for data analysis (Table 3 ). 
Table 3

List of themes, major codes and code titles

Themes

Experience of

previous

caesarean

section

\section{Major codes}

Significance of experience related to previous caesarean section is highlighted by pregnant and delivered women.

Satisfactory experience of previous caesarean section mostly reduces the fear of complications among women for the next caesarean.

First experience of caesarean section sets the precedent for future caesarean sections.

Delivery-related experiences shared by other women

Caesarean sections as a family trend

Caesarean sections as a status symbol

Fear of vaginal childbirth Delivery experience of other women in relatives or friends influence Inductive the women's perceptions of caesarean sections. Shared experiences of other women highlighted caesarean sections Inductive as the less painful childbirth procedure.

In some cases, the complications and pain in wound after

Inductive caesarean surgery becomes more severe than the normal vaginal childbirth.
Caesarean section was considered as a family trend by the women participants.

Caesarean sections are (sometimes) related to body tendency in family.

If mother and sisters have delivered through caesarean section then Inductive the participants believed that they would have a caesarean section as well.

Choosing caesarean surgery as mode of delivery is considered as a status symbol (particularly among rich women) that influences the decision of pregnant women regarding the mode of childbirth.

Women belonging to higher socio-economic status sometimes select caesarean section to assert their social status even in the absence of any medical justification.

Complications during vaginal childbirth process and fear of labour pain are the key reasons for preferring caesarean over vaginal

Code title

Deductive Inductive

Deductive

Inductive

Deductive

Deductive Inductive

Deductive childbirth.

Non-cooperative behaviour of medical staff in labour rooms Inductive increases the fear of vaginal childbirth.

Lack of awareness about modes of delivery
Gynaecologists' opinion and inclination towards caesarean section Inductive play a pivotal role in shaping up the perceptions of pregnant women.

Expectant mothers' inadequate knowledge about childbirth process is likely to increase the chances of their exploitation for economic gains by the gynaecologists. 
The study was approved by the Institutional Ethics Committee of University of the Punjab, Lahore, Pakistan. Confidentiality of the participants and their views was ensured. The researchers took permission from the administration of the respective hospitals. Consent of the women and gynaecologists was taken with the help of informed consent form. Considering the nature of the study, it was essential to select the participants involving their own will and informed consent. For this purpose, the women and gynaecologists were informed about the objectives of the current study and their rights for the participation. All the interviews were conducted at hospitals keeping in view the convenience of the participants. It was ensured that no hospital staff was present during the interviews of women to ensure the privacy of both the interviewer and the interviewees. While informing about their rights to participate in the study, it was mentioned that they may quit an interview if they do not feel comfortable with the nature of the questions asked. However, none of the interviewees left during the interview. In order to ensure the data confidentiality during the process of data collection, information acquired from the participants was strictly concealed from the hospital staff.

\section{Results}

To facilitate the analysis, the findings of the study were analysed under the following major themes: experience of previous caesarean section, delivery-related experiences shared by the other women, caesarean sections as a family trend, caesarean sections as a status symbol, the fear of (prolonged labour during) vaginal childbirth, and lack of awareness about modes of delivery.

\section{Experience of previous caesarean section}

The study found that the beliefs of women are mostly constructed in the context of their personal experiences of previous childbirth. The previous childbirth experiences, either vaginal or caesarean section, usually influence the perceptions of women about the next childbirth. The majority of female participants opined that a satisfactory previous experience of caesarean section reduces the fear of postoperation complications and, thus, a woman does not hesitate to opt for caesarean section in next delivery as well. One of the pregnant women at National Defence Hospital narrated her experience as follows:

My previous caesarean section was an overall satisfactory experience. After discharge from hospital, my only concern was the surgical wound. But after the removal of stitches, everything was fine. So, I am not worried in case I deliver through caesarean again; I would rather like to go for a caesarean section for this delivery as well.

Furthermore, the study participants believed that a caesarean section at first childbirth increases the chances of caesarean sections for their next deliveries as well. Therefore, the women who underwent the caesarean section for the first time considered it unsafe to deliver through vaginal childbirth for the next babies. For example, one of the pregnant women narrated that: 
For my first baby, I was hopeful to have a vaginal childbirth, but I ended up with caesarean section. I would like to have a caesarean section for my second baby, too. I think it will not be safe for me to have a vaginal childbirth after the caesarean section.

\section{Delivery-related experiences shared by other women}

The majority of participants, including gynaecologists as well as delivered and pregnant women, stated that the delivery experiences of other women (relatives or friends) play a significant role in developing their own positive or negative perceptions about the mode of delivery. One of the gynaecologists said that:

Due to lack of awareness about the mode of delivery, many women in our society make up their mind to have a vaginal or caesarean delivery in the light of other women's experiences around them.

When the participants were asked about their perceptions of caesarean section, they spontaneously mentioned their negative or positive expectations associated with this mode of childbirth. A few of the pregnant women highlighted certain advantages of caesarean sections in the light of experiences shared by other women in their social circle. The mostly highlighted advantages of caesarean sections included the lack of labour pain, maintenance of virginal beauty after childbirth, avoidance of numerous internal painful examinations, lack of excessive pressure to the abdomen, and less chances of uterine and ovarian dysfunction. One of the delivered women highlighted that:

Consulting other women during pregnancy is very useful. My colleagues' experience to give childbirth through caesarean section was painless and satisfactory. So, I was not fearful of any complications associated with the caesarean surgery.

Conversely, a few of the pregnant women mentioned myths and negative experiences shared by other women regarding caesarean sections that mostly included the post-surgery pain. Similarly, one of the gynaecologists stated:

Although caesarean sections save women from severe pains of vaginal labour, the pain in the wound after caesarean surgery sometimes becomes more severe than the normal vaginal childbirth.

\section{Caesarean sections as a family trend}

Delivery experiences of other women, especially mother, sisters, aunts and cousins, are considered as a factor significantly influencing the perceptions of women about caesarean section. Interestingly, many participants were of the view that the problems faced by the women in their family during childbirth would eventually be faced by them as well. Few of the pregnant women shared that caesarean section was considered as a family trend and related it to body tendency in their family. For example, one of the pregnant women highlighted that:

My mother had caesarean deliveries and my elder sister could not have vaginal childbirth either. So, I think it is in our family that we cannot have normal vaginal childbirth. 


\section{Caesarean sections as a status symbol}

Caesarean section is an expensive method for childbirth that is affordable for the women belonging to higher socio-economic class. Thus, the selection of mode of delivery tends to divide the Pakistani women implicitly into different social strata in society. Therefore, in many cases, women belonging to higher socio-economic status select this mode of childbirth as a status symbol. A few of the study participants considered it as one of the important factors behind preferring caesarean sections over vaginal childbirth. One of the women living in a posh area of Lahore city stressed that:

Vaginal birth is very painful and it is a nightmare for me to push a baby through vagina. Obviously, I will choose for less painful caesarean section, as I can easily afford it.

While describing the reasons of elective caesarean sections by the women belonging to higher socioeconomic class, one of the gynaecologists informed that:

Women visiting this hospital mostly come from rich families who prefer caesarean section for two main reasons: Firstly, it is less painful and secondly, they consider caesarean an expensive and modern mode of delivery.

\section{Fear of vaginal childbirth}

Fear of vaginal childbirth preoccupies the minds of several pregnant women. The majority of participants expressed fear of prolonged labour pain as the most important factor influencing their perceptions of the mode of delivery, as is visible in a statement by pregnant women:

My friend told that her baby was very healthy, maybe even to strong so that she went through a tough labour experience. I have fear in my mind that it will be hard for me to provide childbirth through vagina. To avoid the bad memories of vaginal childbirth, I prefer a caesarean section.

Another factor highlighted by the majority of participants was the lack of support and cooperation by the medical staff during labour that created fear among women about normal vaginal childbirth. The participants shared the opinion that the non-cooperative and rude behaviour of the medical staff during severe prolonged labour pain added to the anxiety of other pregnant women related to vaginal childbirth. A pregnant woman said:

The labour room environment, especially the behaviour of nurses, is very important. The loud cries and screams due to labour pain create fear and stress among the pregnant women. So, out of this fear, women prefer caesarean section if they can afford it.

\section{Lack of awareness about modes of delivery}

The study found that owing to a lack of awareness about safe modes of delivery and post-delivery complications related to caesarean sections, many women prefer the latter over vaginal childbirth. One of the pregnant women stated: 
During my first childbirth, I opted for caesarean section considering it an easy way to deliver a baby. However, I was not really aware of the post-operation complications. I experienced severe pain in the wound and faced difficulty in performing my routine activities. Therefore, I think it wasn't a wise decision at all.

Furthermore, in the absence of knowledge about mode of delivery, the gynaecologists' opinion tends to become more important in shaping up women's perceptions in favour of caesarean section. Few of the study participants opined that the doctors' preference for caesarean section sometimes involves financial incentives. This seems to be relevant especially in the private hospitals where there is a high cost of caesarean surgery as compare to vaginal childbirth. One of the study participants was of the view that:

I think gynaecologists, mostly in private hospitals, prefer caesarean over vaginal delivery as it involves higher costs and expenses and provide them greater chances to earn more money.

While expectant mothers' inadequate knowledge about childbirth process is likely to increase the chances of their exploitation for economic gains, the participants who were well-informed about the pregnancy complications and the modes of delivery appeared to have lesser chances of exploitation for financial benefits. However, it is important to mention that there were no statements about the misuse of caesarean incision for financial benefits by the gynaecologists, since it was not compulsory for a gynaecologist to provide medical justification for a caesarean section.

\section{Discussion}

In the backdrop of the steady increase in caesarean sections in Pakistan, the present study aimed to investigate different social and cultural aspects highlighted by pregnant and delivered women and gynaecologists that influence the preference for caesarean section over vaginal childbirth. As per the findings of the present study, the fear of prolonged labour during vaginal childbirth, less cooperative behaviour of the medical staff in labour rooms, experience of previous caesarean sections, deliveryrelated experiences shared by the other women, and lack of awareness about mode of delivery were the significant factors that reinforce the positive perceptions of caesarean sections among Pakistani women.

One of the major findings of our study showed that the fear of prolonged labour pain and noncooperative behaviour of nurses during vaginal births create anxiety among women that resultantly influence their perceptions in favour of caesarean section. Correspondingly, the findings of previous studies indicated that pregnant women mostly opt for caesarean section considering it less painful as compared to vaginal childbirth that is accompanied by painful experience of prolonged and uncertain duration of labour hours $[19,20]$. A study conducted in Iran highlighted that the fear of painful labour and lack of supportive behaviour of medical staff in labour rooms are the important factors that reinforce the perceptions of women about caesarean section as a convenient mode of delivery. The study also depicted that women sometimes assume that vaginal pain might lead to vaginal infections in later life and thus they prefer caesarean sections [21]. Similarly, the results of another study highlighted different reasons related to the increasing preference of caesarean section by the women that included socio- 
cultural preferences, avoidance of labour pain, and decreased risk to maternal and fetal life during the childbirth process [22].

The present study found that a satisfactory previous experience of caesarean section reduces the fear of post-operation complications. Thus, pregnant women did not hesitate to opt for caesarean section in their next delivery as well. Similarly, the findings of the previous studies showed that women's perception of caesarean section was influenced by their personal experience of previous childbirth. Women who experienced a high level of satisfaction during a caesarean surgery preferred it again for the next deliveries [2,23]. Previous research indicates that the experience of a woman related to caesarean section is mostly affected by their relationship and communication with their consultant gynaecologist, postoperative care by the hospital staff, and the recovery of wound stitches. All of these factors tend to reinforce the positive perceptions of women about caesarean sections [24].

Additionally, the experience of other women in the closer social network of pregnant women is found to be a key factor influencing their choices regarding the mode of delivery [25]. In correspondence with previous studies [25-27], our study highlighted that pregnant woman decided about caesarean sections in the light of satisfactory delivery experiences of other women. The participants of our study stated that perceptions of caesarean sections were influenced by their observations of other women's delivery and the narration of their post-delivery experiences.

Previous studies showed that the majority of women across the globe are not well-informed about delivery procedures and complications related to caesarean sections [28]. Similarly, the findings of a study conducted in Iran revealed that women who had less awareness regarding disadvantages of caesarean sections preferred it as a mode of delivery [2]. In the same way, it has been shown that a lack of awareness among women regarding pregnancy and delivery complications mostly results in the decision favouring a caesarean section [29]. The present study also highlighted that due to a lack of awareness about post-surgery complications, women opted for caesarean considering it as a less painful delivery procedure. Our study furthermore shows that the lack of awareness among women regarding the childbirth process tends to increase the chances of their exploitation for economic gains. A previous study revealed that gynaecologists were considered as the most important person around a pregnant woman whose informal advice and opinion largely influence their perceptions [30]. Similarly, the findings of the present study suggest that women's perceptions in favour of caesarean section were greatly shaped up in the light of gynaecologist's preference for caesarean sections.

Social perceptions and societal norms significantly affect the decision-making process of women related to the mode of delivery [30]. While the caesarean section is considered prestigious due to higher economic status attached to it, rich women usually prefer it as a mode of delivery [31,32]. Our study also revealed that rich women mostly considered caesarean as an indirect indicator of high socio-economic class and opted it even without any medical reason in order to assert their social class.

\section{Limitations}


In order to gain in-depth understanding of the issue, pregnant women, delivered women, and gynaecologists were included as a sample for this qualitative study. As per our knowledge, no other research has been carried out to understand Pakistani women's perceptions about the upsurge of caesarean section rate. The findings of the study attracted the attention towards the non-medical reasons of caesarean in private hospitals. However, while the gynaecologists were not bound to provide any medical justification for a caesarean section, the study could not provide any evidence about the misuse of caesarean incision for financial benefits by the gynaecologists. Moreover, while the study was limited to an urban sample of female participants and used qualitative approach for data collection, the findings cannot be generalised to the total population.

\section{Conclusion}

Presently, there is an upsurge in the rate of caesarean deliveries all over the world. In Pakistan, the current caesarean rate is more than $19.6 \%$. As per the findings of the present study, the fear of prolonged labour during vaginal childbirth, less cooperative behaviour of the medical staff in labour rooms, experience of previous caesarean section, delivery-related experiences shared by other women, and lack of awareness about mode of delivery were the significant factors that reinforce the positive perceptions of caesarean sections among Pakistani women. The study suggests that the awareness about coping skills to deal with fear and anxiety related to pregnancy and modes of delivery can possibly decrease the caesarean section rate in Pakistan. Additionally, the doctors, especially in private hospitals, may be asked to provide medical justification for carrying out caesarean sections in order to reduce the chances of unnecessary caesarean sections for economic benefits.

\section{Declarations}

\section{Ethical Approval and Consent to Participate}

The Advanced Studies and Review Board, and Doctoral Programme Coordination Committee, University of the Punjab Lahore (Pakistan), provided ethical approval for this study. Written informed consent was obtained from respondents before starting the interviews. Respondents were informed about their rights for volunteer participation. Moreover, the researcher also ensured the confidentiality and anonymity of participants, and their responses.

\section{Consent for Publication}

Not applicable.

\section{Availability of Data and Materials}

Data is available from authors upon reasonable request.

\section{Competing Interest}


The authors declare that no competing interest exist. FF serves on the Editorial Board of BMC Women's Health as Associate Editor.

\section{Funding}

This research received no supporting funds from any funding agency in the public, commercial, or not-forprofit sector.

\section{Authors Contributions}

The study was conceptualized by AJ. AA conducted data collection, AA, AU, FSA, MF and NS were responsible for data analysis, AJ supervised this process. AA drafted the manuscript, AJ and FF revised it critically for important intellectual content. All authors have read and approved the manuscript.

\section{References}

1. Brites R, Nunes O, Pires M, Hipólito J. The Subjective Experience of Pregnancy and the Expectations of Childbirth on a Medicalized World: A Qualitative Study. The Psychologist. 2020;3(1).

2. Latifnejad-Roudsari R, Zakerihamidi M, Merghati-Khoei E, Kazemnejad A. Cultural perceptions and preferences of Iranian women regarding cesarean delivery. Iranian Journal of Nursing Midwifery Research. 2014;19(Suppl.1):28.

3. Torloni MR, Betrán AP, Montilla P, Scolaro E, Seuc A, Mazzoni A, Althabe F, Merzagora F, Donzelli GP, Merialdi M. Do Italian women prefer cesarean section? Results from a survey on mode of delivery preferences. BMC Pregnancy Childbirth. 2013;13(1):78.

4. Walker R, Turnbull D, Wilkinson C. Increasing cesarean section rates: exploring the role of culture in an Australian community. Birth. 2004;31(2):117-24.

5. Bailey P, Lobis S, Maine D, Fortney JA. Monitoring emergency obstetric care: a handbook. Geneva: World Health Organization; 2009.

6. Harrison MS, Saleem S, Ali S, Pasha O, Chomba E, Carlo WA, Garces AL, Krebs NF, Hambidge KM, Goudar SS, Dhaded SM. A Prospective, Population-Based Study of Trends in Operative Vaginal Delivery Compared to Cesarean Delivery Rates in Low- and Middle-Income Countries, 2010-2016. Am J Perinatol. 2019;36(7):730-6.

7. National Institute of Population. Studies (NIPS) [Pakistan], ICF. Pakistan Demographic and Health Survey 2017-18.

8. Garmaroudi GR, Eftekhar H, Batebi A. Factors that affect cesarean among pregnant women. Payesh J. 2002;1(2):45-9.

9. Buekens P. Over-medicalisation of maternal care in developing countries. In: De Brouwere V, Van Lerberghe W, editors. Safe motherhood strategies: a review of the evidence. Antwerp: ITGPress; 2001. pp. 195-206. 
10. Amjad A, Amjad U, Zakar R, Usman A, Zakar MZ, Fischer F. Factors associated with caesarean deliveries among child-bearing women in Pakistan: Secondary analysis of data from the Demographic and Health Survey, 2012-13. BMC Pregnancy Childbirth. 2018;18(1):113.

11. Lei H, Wen SW, Walker M. Determinants of caesarean delivery among women hospitalized for childbirth in a remote population in China. Journal of Obstetrics Gynaecology Canada. 2003;25(11):937-43.

12. Gholami A, Salarilak S. Why do some pregnant women prefer cesarean delivery in first pregnancy? Iranian Journal of Reproductive Medicine. 2013;11(4):301.

13. Dantas JA, Singh D, Lample M. Factors affecting utilization of health facilities for labour and childbirth: a case study from rural Uganda. BMC Pregnancy Childbirth. 2020;20(1):39.

14. Howell S, Johnston T, Macleod SL. Trends and determinants of caesarean sections births in Queensland, 1997-2006. Aust N Z J Obstet Gynaecol. 2009;49(6):606-11.

15. Lori JR, Boyle JS. Cultural childbirth practices, beliefs, and traditions in post-conflict Liberia. Health Care Women Int. 2011;32(6):454-73.

16. Abbaspoor Z, Moghaddam-Banaem L, Ahmadi F, Kazemnejad A. Iranian mothers' selection of a birth method in the context of perceived norms: A content analysis study. Midwifery. 2014;30(7):804-9.

17. Popham J. Difficult Questions for Difficult Decisions: A Book Review. The Qualitative Report. 2019;24(7):1782-3.

18. Silverman D. What counts as qualitative research? Some cautionary comments. Qualitative Sociology Reviews. 2019;9(2):48-55.

19. Adams SS, Eberhard-Gran M, Eskild A. Fear of childbirth and duration of labour: a study of 2206 women with intended vaginal delivery. International Journal of Obstetrics Gynaecology. 2012;119(10):1238-46.

20. Uçar T, Golbasi Z. Effect of an educational program based on cognitive behavioural techniques on fear of childbirth and the birth process. Journal of Psychosomatic Obstetrics Gynecology. 2019;40(2):146-55.

21. Movahed M, Enayat H, Ghaffarinasab E, Alborzi S, Mozafari R. Related factors to choose cesarean rather than normal delivery among Shirazian pregnant women. Journal of Fasa University of Medical Sciences. 2012;2(2):78-83.

22. Sluijs AM, Wijma K, Cleiren MP, van Lith JM, Wijma B. Preferred and actual mode of delivery in relation to fear of childbirth. Journal of Psychosomatic Obstetrics Gynecology. 2020;2:1-9.

23. Dodd JM, Crowther CA, Huertas E, Guise JM, Horey D. Planned elective repeat caesarean section versus planned vaginal birth for women with a previous caesarean birth. Cochrane Database of Systematic Reviews. 2013;12:CD004224.

24. Fumagalli S, Colciago E, Antolini L, Perego S, Fiorasi M, Fossati V, Nespoli A, Vergani P. Women's decision-making about mode of birth after a previous caesarean section. British Journal of Midwifery. 2020;28(2):104-13. 
25. Nisar N, Sohoo NA, Memon A. Knowledge, attitude and preferences of pregnant women towards modes of delivery. Journal of the Liaquat University of Medical Health Sciences. 2009;8(3):228-33.

26. Zakerihamidi M, Roudsari RL, Khoei EM. Vaginal delivery vs. cesarean section: A focused ethnographic study of women's perceptions in The North of Iran. International Journal of Community-based Nursing Midwifery. 2015;3(1):39.

27. Buyukbayrak EE, Kaymaz O, Kars B, Karsidag AYK, Bektas E, Unal O, Turan C. Caesarean delivery or vaginal birth: preference of Turkish pregnant women and influencing factors. J Obstet Gynaecol. 2010;30(2):155-8.

28. Oliveira RR, Melo EC, Novaes ES, Ferracioli PL, Mathias TA. Factors associated to caesarean delivery in public and private health care systems. Revista da Escola de Enfermagem da USP. 2016;50(5):733-40.

29. Agarwal A, Saxena R, Barabanki UP. Evaluation of postoperative complications in patients undergoing Cesarean section: An observational study. Journal of Advanced Medical Dental Sciences Research. 2019;7:8.

30. Weaver JJ, Statham H, Richards M. Are there "unnecessary" cesarean sections? Perceptions of women and obstetricians about cesarean sections for nonclinical indications. Birth. 2007;34(1):3241.

31. Pai M. Unnecessary medical interventions: caesarean sections as a case study. Economic Political Weekly. 2000;35(31):2755-61.

32. Karlström A, Nystedt A, Johansson M, Hildingsson I. Behind the myth-few women prefer caesarean section in the absence of medical or obstetrical factors. Midwifery. 2011;27(5):620-7.

\section{Supplementary Files}

This is a list of supplementary files associated with this preprint. Click to download.

- AppendixInterviewguides.pdf 